\title{
IT ISN'T ADDING UP: THE GAP BETWEEN THE PERCEPTIONS OF ENGINEERING MATHEMATICS STUDENTS AND THOSE HELD BY LECTURERS IN THE FIRST YEAR OF STUDY OF ENGINEERING
}

\author{
Ken Brown ${ }^{1}$, Victor Lally ${ }^{2}$ \\ ${ }^{1}$ Letterkenny Institute of Technology (IRELAND) \\ ${ }^{2}$ University of Glasgow (UNITED KINGDOM)
}

\begin{abstract}
The transition from second level to third level education offers a considerable degree of challenge for many students. One such challenge is the students' internalized world-view and its effects on the potential of the students. The perceptions, self-efficacy, and expectancy, of the students as they move into third level education, may be significantly at variance with the beliefs held by lecturers. This research project began as an individual case study of first year engineering students in one higher education organization and was extended to include a similar organization in another EU country. Through a series of questionnaires and group interviews with students the thematic outputs of the process formed the basis for questioning of lecturers. Evidence from the data revealed many students in the initial stages of the first-year of study display low levels of confidence, do not understand the higher education assessment processes, and perceive many barriers to progress. Analysis of individual lecturer interviews shows that many do not share assessment data with students. In an attempt to ascertain if the issues raised are localized, further research has begun to gather data from students in similar Higher Education organisations in an additional four countries. The outcomes suggest an alternative approach to assessment of engineering mathematics is required.assist you in formatting your paper. Please, insert the text keeping the format and styles. The parts of the paper (title, abstract, keywords, sections, text, etc.) are already defined on the style sheet, as illustrated by the portions given in this document.
\end{abstract}

Keywords: First-Year Experience, Self-Efficacy, Expectancy, Online Assessment, Mathematics.

\section{INTRODUCTION}

A major concern for many higher education institutions is the increased diversity of mathematical skills (Kinnari, 2010; Gallimore \& Stewart, 2014; Prendergast, Breen, Carr \& Faulkner, 2016) and decline in mathematical skills (Treacy \& Faulkner, 2015) offered by students on entry to first year engineering programmes in Ireland. Cole et al (2014) outlines the difficulties experienced in the teaching of mathematics to first year engineering students as a direct result of the wide range in students' mathematical ability in a United Kingdom university. A comparison is made with the Finnish education system where approximately $55 \%$ of second level students study a mathematics programme (Rinneheimo, 2010) compared to typically 13\% in the United Kingdom (OECD, 2013). The literature concentrates on students at university - they may be regarded as high educational achievers at second level. This study focuses on low-to-medium educational achievers and there is a paucity of literature exploring such student types.

Regarding students in Ireland, an engagement with Technology Enhanced Learning (TEL) would be considered atypical for the majority at second level. The delivery paradigm is face-to-face teaching combined with structured high-value end of programme examinations. The students are not prepared for the delivery paradigms offered by Higher Education (McCraith 2015). The Higher Education model expects the student to be a motivated, self-directed learner, and be fully cognizant of digital learning tools. Self-directed learners display metacognitive traits (Robles \& Braathen, 2002; Schneider \& Artelt, 2010) as they monitor and self-regulate their behaviour and this is typical of students from higher academic tracks; they have established proven cognitive and metacognitive strategies. The sociocognitive environment is crucial to the promotion of the amplification of the cognitive abilities of the cognitive apprentice in terms of their metacognition and associated behaviours.

Entry to third level for low-to-medium educational achievers carries extra risks compared to higher educational achievers. A new paradigm of education in the form of large lectures, tutorials and, assessments, combined with TEL is now thrust at these students for the first time. Universities report drop out rates and lack of retention as serious issues to be dealt with and yet low-to-medium 
achievers are expected to cope to the same degree. The appropriate pedagogical application of new technologies within TEL programmes of study has been identified as core to the perceived success of such programmes. As programmes expand beyond the enclosed environments of Higher Education into the public domain, via distance learning, the role of the new technologies becomes not just pivotal to the success of the programmes but is also a major affecting element for students. Digital literacy and skills have been identified within many studies as being critical to the success of both students and their programmes of study.

The Higher Education domain utilizes Computer Aided Assessment widely where many see this as a means of coping with the pressures of assessing large class groups in a fast, efficient manner. Speed of return for feedback (Torrance, 2012) - even though some students choose to ignore the feedback in particular is viewed as critical and increasingly there is a need for personalized feedback (Narciss et al, 2014) as opposed to generalized feedback. Jordan (2013) considered the growing maturity of eassessment systems for mathematics suggesting that such systems may become generally available. The main technique for the assessment of mathematics being addressed in contemporary literature is that of Computer Algebra Systems (Sangwin, 2012; Carrol et al, 2016) offering the capability of assessing beyond simple questioning such as Multiple Choice, and basic calculation (Gallimore \& Stewart, 2014). The majority of studies in the TEL related assessment literature are practitioner based (Whitelock, Gilbert \& Gale, 2013), hence there is a certain degree of difficulty in determining suitability of particular assessment approaches.

The research questions being addressed within this study are:

- Are students prepared for online assessment of mathematics in the first year of study in higher education?

- Do students perceive barriers that may form impediments to online assessment of mathematics in the first year of study in higher education?

- Does the self-efficacy of the students affect the perceptions of students with respect to online assessment of mathematics?

- What level of understanding of assessment and feedback do the students hold?

- What perceptions do instructors have in the first year of third level engineering programmes?

To address these questions a research study was designed using a social-cognitive approach bounded by self-efficacy theory within the domain of engineering mathematics (Bandura, 1977; Martchand \& Gutierrez, 2012; Artino, 2012). Engineering mathematics is the focus of the research as the researchers attempt to obtain an improved understanding of the online experiences of the students as they progress through their first year of study in engineering. Self-efficacy posits that the observations and actions of the students influence their subsequent actions and reactions. Hence, prior or pre-existing attributes are considered to be determining factors of self-efficacy and awareness of learning. However, the students do not exist in a state of isolation at third level due to the interaction of lecturers (Temple \& Newmann, 2014). The study explores the perceptions and beliefs that lecturers hold in relation to the students to determine if there is any mismatch between lecturers and students.

\section{METHODOLOGY}

\subsection{Learner Groups}

A mixed methods approach was designed to integrate and guide the initial outputs with qualitative and quantitative approaches operating in close synchronicity. Each participant engaged with their consent by completing an anonymized questionnaire containing open and closed questions.

Discussion group sampling activity was based on convenience by the availability of learners to the researcher. Non self-selecting groups from Ireland were drawn from the group of available first year participants within a standard timetabled session. Second year self-selecting student sample groups made themselves available during a lunch time session during semester 4 . The students from Finland were self-selecting and participated in a non-timetabled session whilst speaking in English. Discussion group activities utilized a semi-structured, standardized open-question approach, timed to take place shortly after operationalization of the questionnaire and immediately after the first online assessment. All interviewees were asked the same basic questions to ensure comparability of responses. 


\subsection{Lecturer Group}

The study involved mathematics lecturers from Ireland, Finland, and United Kingdom. Each lecturer participated with consent in an anonymized semi-structured video interview and was asked the same questions to allow comparisons to be made. Prior to the lecturer interviews an analysis of the student questionnaires was conducted to establish main thematic areas for consideration. The selected thematic areas were deduced from the completed student questionnaires using the combination of responses to open and closed questions.

\subsection{Analysis}

\subsubsection{Quantitative Instruments}

The questionnaires in the first phase piloted in Ireland and Finland prior to delivery and contained a total of 7 questions, several multiple-choice, six-point Likert-scale matrix, and open-ended questions. The complete data set was entered into SPSS for analysis. Open-ended responses were revisited to confirm the validity of the codes generated from the analysis. The themes were compared with the research questions to determine their appropriateness and to ascertain if the questions needed to be re-evaluated.

The questionnaires in the second phase were piloted in Ireland, Finland, and Poland, prior to delivery and contained a total of 16 questions. The first two questions relate to gender and prior knowledge of computer based testing. The remaining fourteen questions are six-point Likert scale based relating to confidence, self-efficacy and expectancy. The questionnaire was also translated to Russian and Estonian to address eastern European students.

\subsubsection{Qualitative Instruments}

Qualitative analysis of the questionnaires and video interviews used Interpretative Phenomenological Analysis (Symeonides \& Childs, 2015). The engagement is a hermeneutic approach where transcriptions are viewed as textual representations of idiographic experiences to establish a baseline of the status of online assessment experiences of first year learners in engineering mathematics. A coding schema (Ryan \& Bernard, 2003) was developed and the level of granularity for the analysis was determined to be an utterance - all utterances are considered unique within this research. The code selected to represent the utterance would not change until a succeeding utterance, response, or phrase required an alternative code.

\section{RESULTS}

Four major factors have emerged: Confidence, Feedback, Self-efficacy and Expectancy. 262 students completed the questionnaires in phase one and 53 students participated in the group interviews in Ireland and Finland. $69.1 \%$ indicated prior experience of computer based testing with $32.8 \%$ noting a negative experience, $84.2 \%$ group indicated moderate confidence or higher. $33.4 \%$ of student utterances related to confidence - both negative and positive. Confidence utterances dropped to $31.7 \%$ in year 2 but the proportion of negative confidence comments increased to $70 \%$ and was closely related to issues of feedback. $5.22 \%$ of first year student comments are related to feedback rising to $18.5 \%$ by semester 4 in the second year of study.

[S6_Y2, Dec 2016] "But there is no feedback ..."

Lecturers mentioned feedback $5.8 \%$ of the time whilst discussing assessment; the comment by Student6_Y2 is not invalid.

$[$ [ 9, 2016] "I didn't really give feedback. It was just a mark allocated".

Utterances by seven lecturers indicated they felt students did not have any problems with confidence. Two lecturers expressed awareness of issues of lack of confidence in mathematics. $56.2 \%$ of utterances of self-efficacy were positive in nature in first semester mathematics.

The perceived valence placed on the outcome by the student is a source of motivation affecting selfefficacy. $72 \%$ of first year student utterances related to feelings of negative expectancy due to the lack of partial credit in grading. Positive self-efficacy drops to $6.1 \%$ and negative expectancy drops to $20.3 \%$ and negative confidence accounts for $31.7 \%$ of utterances in year 2 . Students are concerned about a relative reduction in feedback within the mathematics programme in year 2 in comparison with 
year $1.57 .7 \%$ of utterances by lecturers in relation to their perceptions of self-efficacy in students are positive. $37 \%$ of expectancy utterances are negative i.e. there is a belief that the performance of students is not valued. The extra effort expended completing online assessments is discussed and considered to have a negative impact:

[F3_Y1_Fi, 2017] "Oh God, not again! Can I do it another way?"

[F4_Y1_Fi, 2017] "With mathematics it would be nice if we had a way to write online in the traditional way."

Phase two of the questionnaire process is currently underway and an initial analysis is expected to be available shortly.and limit the use of hard returns to one return at the end of a paragraph.

\section{CONCLUSIONS}

Two cohorts of students have progressed through the study since academic year 2015/16 in Ireland and Finland. The responses to the initial questionnaires and subsequent comments made within the interviews are generally in agreement. The feelings of low confidence in the Irish students is repeated as is the high confidence levels of the Finnish students in relation to their abilities in mathematics and both sets experience high levels of anxiety in relation to online assessment. The access to TEL at second level is low according to both sets of students, however the post experience comments are relatively positive for the Irish students. A degree of anxiety is visible in the interview outputs; the reasons for the anxiety adjust as the students move through the programme of study and leads to issues in second year of study. The lack of familiarity with e-Assessment is one of the largest hurdles for students to overcome in both countries. Some students grow to enjoy e-Assessment whilst others literally hate the process. A familiar phrase used is that e-assessment of mathematics is not like using a pen and paper - it is a foreign language. The term feedback is not widely used by first year students until the beginning of second semester - the assessment process is not fully understood by many in the initial stages of the first year of study.

\section{ACKNOWLEDGEMENTS}

The authors wish to thank Mr. Jarkko Hurme, Oulu University of Applied Sciences, for his invaluable support in this study.

\section{REFERENCES}

[1] Kinnari, H., (2010). A study of the mathematics proficiency. $1^{\text {st }}$ intl workshop on maths and ICT: Education, Research and Applications, Bucharest, 35-39.

[2] Gallimore, M. \& Stewart, J., 2014, Increasing the impact of mathematics support on aiding student transition in higher education, Teaching Mathematics and its Applications, 33(2), pp. 98109.

[3] Prendergast, M., Breen, C., Carr, C. \& Faulkner, F., 2016, Investigating Third Level Lecturers' Awareness of Second level Curriculum Reform, 13th International Congress on Mathematical Education.

[4] Treacy, P., \& Faulkner, F., (2015). Analysing the effects of the introduction of the new Project Maths syllabus on beginning undergraduates' performance of basic mathematical skills in Ireland. $13^{\text {th }}$ Intl Conference of the mathematics education for the future project: Mathematics education in a connected world, Catania, Sicily, Italy.

[5] Cole, J. S., McCartan, C.D., Tuohi, R., \& Steinby, P.,(2014). Mathematics Background of Engineering Students in Northern Ireland and Finland. Proc of the 10th Int/ CDIO Conference, Universitat Politecnica De Catalunya.

[6] Rinneheimo, K., (2010). Methods for teaching mathematics case Tampere University of Applied Sciences. $1^{\text {st }}$ intl workshop on maths and ICT: Education, Research and Applications, Bucharest, 48-55.

[7] OECD, 2013, Impact of the Economic Crisis on Public Education, OECD 2013, [accessed online 24/05/16] https://www.oecd.org/edu/skills-beyond-school/EDIF\%202013-No18\%20(eng).pdf 
[8] McCraith, B., (2015). Average is no longer good enough - it's time for a step change in STEM education in Ireland. In Education Matters, Yearbook 2016, 13-18.

[9] Robles, M. \& Braathen, S., 2002, Online assessment techniques, Delta Pi Epsilon Journal, 44(1), pp. $39-50$.

[10] Schneider, W. \& Artelt, C., 2010, Metacognition and mathematics education, ZDM, 42(2), pp. 149-61.

[11] Torrance, H., 2012, Formative assessment at the crossroads: conformative, deformative and transformative assessment, Oxford Review of Education, 38(3), pp. 323-42.

[12] Narciss, S., Sosnovsky, S., Schnaubert, L., Andrès, E., Eichelmann, A., Goguadze, G. \& Melis, E., 2014, Exploring feedback and student characteristics relevant for personalizing feedback strategies, Computers \& Education, 71, pp. 56-76.

[13] Jordan, S., 2013, E-assessment: Past, present and future, New Directions, 9(1), pp. 87-106.

[14] Sangwin, C., 2012, Computer Aided Assessment of Mathematics using STACK, $12^{\text {th }}$ International Congress on Mathematical Education.

[15] Carroll, T., Casey, D., Crowley, J., Mulchrone, K., \& Ni She, A., 2016, EAssessment in Mathematical Sciences, Numbas as an engagement tool for first year Business Studies students. Conf. E-Assessment in Mathematical Sciences, Newcastle [accessed online: 04/11/16] www.teame.ie/wp-content/uploads/2016/10/Newcastle-paper.pdf.

[16] Whitelock, D., Gilbert, L., and Gale, V., 2013, e-Assessment tales - what types of literature are informing day-to-day practice?, Report to Higher Education Academy, [accessed online: 30/08/15] http://www.researchgate.net/publication/272357064.

[17] Bandura, A., (1977). Self-efficacy: Toward a Unifying Theory of Behavioral Change. Psychological Review, 84(2), 191-215

[18] Marchand, G.C. \& Gutierrez, A.P., 2012, The role of emotion in the learning process: Comparisons between online and face-to-face learning settings, The Internet and Higher Education, 15(3), pp. 150-60.

[19] Artino Jr, A.R., (2012). Academic self-efficacy: from educational theory to instructional practice. Perspect Med Educ, 1,76-85

[20] Tempel, T. \& Neumann, R., 2014, Stereotype threat, test anxiety, and mathematics performance, Social Psychology of Education, 17(3), pp. 491-501.

[21] Symeonides, R., \& Childs, C., (2015). The personal experience of online learning: An interpretative phenomenological analysis. Computers in Human Behavior, 51, 539-545.

[22] Ryan, G.W., \& Bernard, S., (2003). Techniques to Identify Themes. Field Methods, 15(1), 85109. 